CERN-TH/95-66

hep-ph/9503171

\title{
Derivatives as an IR Regulator for Massless Fields
}

\author{
Per Elmfors' \\ CERN, TH Division, CH-1211 Genève 23, Switzerland
}

\begin{abstract}
The free propagator for the scalar $\lambda \phi^{4}$-theory is calculated exactly up to the second derivative of a background field. Using this propagator I compute the one-loop effective action, which then contains all powers of the field but with at most two derivatives acting on each field. The standard derivative expansion, which only has a finite number of derivatives in each term, breaks down for small fields when the mass is zero, while the expression obtained here has a well-defined expansion in $\phi$. In this way the resummation of derivatives cures the naive IR divergence. The extension to finite temperature is also discussed.
\end{abstract}

${ }^{1}$ E-mail: per.elmfors@cern.ch 


\section{INTRODUCTION}

There are a number of methods for computing approximations to the effective action depending on which parameters can be considered as small. If the field amplitude is small it may be enough to compute a small number of $n$-point functions, with the advantage that they can be computed to all orders in the derivatives. For instance, the two-point function can be calculated to all orders in momentum, yielding corrections to the dispersion relation. For configurations with large fields all $n$-point functions become important and have to be resummed. This we can do for a constant field, thus assuming all derivatives to be zero, and we get the effective potential. Even though the effective potential is calculated for a strictly constant field, the effective action, expanded to the zeroth order in derivatives, is obtained from it by simply replacing the constant by a slowly varying $x$-dependent field. By doing so it is assumed that the field varies so slowly that it can locally be approximated by a constant. Effects that have to do with global properties, such as phase separation in spontaneously broken theories or instanton contributions, are completely neglected in the quantum corrections, although there is no direct assumption that the amplitude of the field variation must be small. In a derivative expansion of the effective action the next step is to calculate the wave function renormalization to all orders in the field without derivatives. The reason why the effective potential and the wave function renormalization can be calculated to all orders in the field from a one-loop calculation is that the propagator can be constructed exactly in the presence of a constant background field. It is, therefore, interesting to see if one can go further in the local approximation while still being able to find the exact propagator. In this paper I use Schwinger's proper-time method [1] to compute the propagator exactly up to the second derivative of the field and use this in turn to compute the effective action and the effective equations of motion.

I restrict the calculations here to a toy model, the $\lambda \phi^{4}$-model, to study general properties of the method. It would be interesting to extend the method to more realistic theories, in particular gauge theories. The effective potential has been used to a large extent in the electroweak model to calculate bubble properties at the phase transition. The wave function renormalization constant $Z(\phi)$ has also been computed [2]. There are two main problems with that procedure. First, it is very sensitive to the choice of gauge fixing parameter, because the usual effective action suffers from gauge dependence away from its stationary points. (The Vilkovisky-DeWitt effective action might be the correct way to get around this problem.) Secondly, $Z(\phi)$ diverges when the field goes to zero because the transverse gauge field is unscreened at high temperature. In this paper I discuss a way to cure the IR sensitivity by including higher derivative terms. The idea is that if the field is much smaller than the scale 
set by the derivative, then the derivative expansion does no longer hold and higher derivatives have to be included exactly. On the other hand, when the derivatives are non-negligible, the field cannot be small in any sizeable volume and in this way the derivatives regulate the IR sensitivity. The approximation is still local and there is no hope that it would cure the imaginary parts that occur in a spontaneously broken theory when the mass squared is negative. It may, therefore, be difficult to apply the method directly to bubble wall calculations in the electroweak theory.

\section{LOCAL EXPANSION OF THE EFFECTIVE ACTION}

The basic equation to use when calculating the effective action is the generalized tadpole equation [3]

$$
\frac{\delta \Gamma[\phi(y)]}{\delta \phi(x)}=\Gamma^{(1)}[\phi(y) ; x]
$$

which gives the relation between the effective action $\Gamma[\phi]$ and the one-point function $\Gamma^{(1)}[\phi ; x]$ computed in the background of $\phi(y)$. To find $\Gamma[\phi]$ one has to integrate $\Gamma^{(1)}[\phi ; x]$ with respect to $\phi(x)$, but the equation of motion is given directly by $\Gamma^{(1)}[\phi ; x]=0$. The Feynman rules needed to compute the one-point function are derived from the Lagrangian in the non-trivial background

$$
\begin{aligned}
\mathcal{L}(\phi)= & \frac{1}{2}\left((\partial \phi)^{2}-m^{2} \phi^{2}\right)-\frac{\lambda}{4 !} \phi^{4} \\
\mathcal{L}(\phi+\eta)= & \mathcal{L}(\phi)+\frac{\delta \Gamma_{c l}}{\delta \phi} \\
& +\frac{1}{2}\left((\partial \eta)^{2}-\left(m^{2}+\frac{\lambda \phi^{2}}{2}\right) \eta^{2}\right)-\frac{\lambda}{3 !} \eta^{3}-\frac{\lambda}{4 !} \eta^{4} .
\end{aligned}
$$

The tadpole equation to one loop is then written as

$$
i \Gamma^{(1)}[\phi ; x]=i \frac{\delta \Gamma_{c l}}{\delta \phi}+\frac{1}{2}(-i \lambda \phi(x))\left\langle x\left|\frac{i}{\hat{p}^{2}-M^{2}(\phi)+i \epsilon}\right| x\right\rangle,
$$

with $M^{2}(\phi)=m^{2}+\lambda \phi^{2}(\hat{x}) / 2$. This equation can be integrated to give

$$
\Gamma[\phi]=\Gamma_{c l}+\frac{i}{2} \operatorname{Tr}_{x} \ln \left(\hat{p}^{2}-M^{2}+i \epsilon\right)
$$

Because of the trace over one-particle states labelled by $x$, there is no ordering problem when doing the integration, even though $\hat{p}$ and $\phi(\hat{x})$ do not commute. From Eq. (5) we identify the one-loop Lagrangian and use the proper-time representation to write it as

$$
\mathcal{L}^{(1)}(x)=-\frac{i}{2} \int_{0}^{\infty} \frac{d s}{s}\left\langle x\left|e^{i s\left(\hat{p}^{2}-M^{2}+i \epsilon\right)}\right| x\right\rangle \equiv-\frac{i}{2} \int_{0}^{\infty} \frac{d s}{s}\langle x(s) \mid x(0)\rangle .
$$


This expression has, of course, the usual UV infinities which we have to renormalize at the end.

In a general background it is not possible to calculate $\left\langle x\left|\exp \left[i s\left(\hat{p}^{2}-M^{2}+i \epsilon\right)\right]\right| x\right\rangle$ exactly since $\left[\hat{p}^{2}, M^{2}(\phi(\hat{x}))\right] \neq 0$. If we think of Eq. (6) as a purely quantum mechanical problem, the non-trivial part of the amplitude from $|x\rangle$ to $\langle x|$ comes from quantum fluctuations. That is, the virtual particle that propagates from $x$ back to $x$ probes a certain neighbourghood of $x$ related to the Compton wavelength. Expanding the background field, i.e. $M^{2}(\phi)$, in derivatives should lead to a good approximation if these fluctuations are not too large. Notice that this is different from a derivative expansion of the amplitude itself, which is a complicated function of $M^{2}$. To second order in derivatives we define

$$
\begin{aligned}
F_{\mu} & =\partial_{\mu} M^{2}=\lambda \phi \partial_{\mu} \phi \\
\omega_{\mu \nu} & =\partial_{\mu} \partial_{\nu} M^{2}=\lambda \partial_{\mu} \phi \partial_{\nu} \phi+\lambda \phi \partial_{\mu} \partial_{\nu} \phi .
\end{aligned}
$$

When computing the Lagrangian at a point $x$ we expand the position operator in $M^{2}(\phi)$ around that point $(x \mapsto x+\hat{x}, \hat{x}|x\rangle=0)$, and define the corresponding bilinear Hamiltonian (suppressing the $+i \epsilon$ term in the sequel)

$$
\begin{aligned}
M^{2}(\phi(x+\hat{x})) & \simeq M^{2}+\hat{x}^{\mu} F_{\mu}+\frac{1}{2} \hat{x}^{\mu} \omega_{\mu \nu} \hat{x}^{\nu}, \\
H & =\hat{p}^{2}-M^{2}-\hat{x} F-\frac{1}{2} \hat{x} \omega \hat{x},
\end{aligned}
$$

using a matrix notation in the second line of Eq. (8). Following Schwinger [1] the problem has now been reduced to solving the differential equation

$$
\begin{aligned}
\frac{d}{d s}\left\langle x^{\prime \prime}(s) \mid x^{\prime}(0)\right\rangle & =i\left\langle x^{\prime \prime}(s)|H| x^{\prime}(0)\right\rangle, \\
\left\langle x^{\prime \prime}(0) \mid x^{\prime}(0)\right\rangle & =\delta\left(x^{\prime \prime}-x^{\prime}\right) .
\end{aligned}
$$

In order to write Eq. (9) as an explicit differential equation, $H(\hat{p}(0), \hat{x}(0))$ has to be rewritten as a function of $\hat{x}(s)$ and $\hat{x}(0)$ using the equations of motion for $\hat{p}$ and $\hat{x}$

$$
\frac{d}{d s}\left(\begin{array}{c}
\hat{x}(s) \\
\hat{p}(s)
\end{array}\right)=-i\left[H(\hat{p}(s), \hat{x}(s)),\left(\begin{array}{c}
\hat{x}(s) \\
\hat{p}(s)
\end{array}\right)\right]=\left(\begin{array}{cc}
0 & 2 \\
\omega & 0
\end{array}\right)\left(\begin{array}{c}
\hat{x}(s) \\
\hat{p}(s)
\end{array}\right)+\left(\begin{array}{c}
0 \\
F
\end{array}\right) .
$$

The solution is

$$
\left(\begin{array}{c}
\hat{x}(s)+\omega^{-1} F \\
\sqrt{\frac{2}{\omega}} \hat{p}(s)
\end{array}\right)=\left(\begin{array}{cc}
\cosh (s \sqrt{2 \omega}) & \sinh (s \sqrt{2 \omega}) \\
\sinh (s \sqrt{2 \omega}) & \cosh (s \sqrt{2 \omega})
\end{array}\right)\left(\begin{array}{c}
\hat{x}(0)+\omega^{-1} F \\
\sqrt{\frac{2}{\omega}} \hat{p}(0)
\end{array}\right),
$$


and the differential equation (9) is explicitly written as

$$
\begin{aligned}
& \frac{d}{d s}\left\langle x^{\prime \prime}(s) \mid x^{\prime}(0)\right\rangle \\
& =\left\{i\left[F^{\prime \prime} \frac{1}{2 \omega} \frac{1}{\sinh ^{2}(s \sqrt{2 \omega})} F^{\prime \prime}+F^{\prime} \frac{1}{2 \omega} \frac{\cosh ^{2}(s \sqrt{2 \omega})}{\sinh ^{2}(s \sqrt{2 \omega})} F^{\prime}-F^{\prime \prime} \frac{\cosh (s \sqrt{2 \omega})}{\omega \sinh ^{2}(s \sqrt{2 \omega})} F^{\prime}\right]\right. \\
& \left.\quad-i s M^{2}\left(\phi\left(x^{\prime}\right)\right)-\operatorname{tr}\left(\sqrt{\frac{\omega}{2}} \frac{\cosh (s \sqrt{2 \omega})}{\sinh (s \sqrt{2 \omega})}\right)\right\}\left\langle x^{\prime \prime}(s) \mid x^{\prime}(0)\right\rangle
\end{aligned}
$$

where we have expanded $M^{2}(\phi)$ around $x^{\prime}$. This choice of expansion point will not matter when we later take $x^{\prime}=x^{\prime \prime}=x$. The notation $F^{\prime \prime}$ and $F^{\prime}$ means $F_{\mu}\left(x^{\prime \prime}\right)$ and $F_{\mu}\left(x^{\prime}\right)$. The non-trivial functions of $\omega$ should be defined by power series expansions in $\omega^{\mu}{ }_{\nu}$ with suitable contractions with $F_{\mu}$. The solution of Eq. (12) in $d$-dimensional Minkowski space is

$$
\begin{aligned}
\left\langle x^{\prime \prime}(s) \mid x^{\prime}(0)\right\rangle= & e^{-i \frac{\pi}{4}(d-2)}(4 \pi s)^{-d / 2} \operatorname{det}\left(\frac{s \sqrt{2 \omega}}{\sinh (s \sqrt{2 \omega})}\right)^{1 / 2} \\
\exp \{i & -F^{\prime \prime} \frac{\operatorname{coth}(s \sqrt{2 \omega})}{(2 \omega)^{3 / 2}} F^{\prime \prime}+F^{\prime}\left(\frac{s}{2 \omega}-\frac{\operatorname{coth}(s \sqrt{2 \omega})}{(2 \omega)^{3 / 2}}\right) F^{\prime} \\
& \left.\left.+F^{\prime \prime} \frac{1}{\sqrt{2 \omega}} \frac{1}{\omega \sinh (s \sqrt{2 \omega})} F^{\prime}-s M^{2}\right]\right\} .
\end{aligned}
$$

Finally, taking $x^{\prime}=x^{\prime \prime}=x$ we find

$$
\begin{aligned}
\mathcal{L}^{(1)}(x)= & \frac{i}{2} \int_{0}^{\infty} \frac{d s}{s}(4 \pi s)^{-d / 2} e^{-i \frac{\pi}{4}(d-2)} \operatorname{det}\left(\frac{s \sqrt{2 \omega}}{\sinh (s \sqrt{2 \omega})}\right)^{1 / 2} \\
& \exp \left\{-i s\left[M^{2}-F \frac{1}{2 \omega}\left(1-\frac{2}{s \sqrt{2 \omega}} \tanh \frac{s \sqrt{2 \omega}}{2}\right) F\right]\right\} .
\end{aligned}
$$

Notice that the integrand is well-defined as a power series in $\omega^{\mu}{ }_{\nu}$ even when $\omega^{\mu}{ }_{\nu}$ is not invertible. The integrand has possible poles on the real and imaginary axes, but only there since eigenvalues of $\omega$ are real. We now want to deform the $s$-contour to the negative imaginary axis. This is possible if the arc at infinity gives a vanishing contribution. In our case we must require $M^{2}>\frac{1}{2} F^{\mu}\left(\omega^{-1}\right)_{\mu \nu} F_{\nu}$. If we assume the eigenvalues of $\omega_{\mu \nu}$ to be positive, the condition on $M^{2}$ is the same as saying that the mass squared, in the quadratic approximation $M^{2}(x+\xi) \simeq M^{2}(x)-F_{\mu} \xi^{\mu}+\frac{1}{2} \xi^{\mu} \omega_{\mu \nu} \xi^{\nu}$, is positive everywhere, not only at $x$. We now assume this condition to be satisfied and perform the contour deformation. If the above condition is not satisfied, we would expect an imaginary part since the particles can then (in the quadratic approximation) propagate to a point where they become tachyonic. Such an imaginary part would be an artefact of the approximation rather than a physical reality if the full $M^{2}(\phi)$ indeed is positive everywhere. 
In 4 dimensions $(d=4)$ Eq. (14) is infinite as it stands and has to be renormalized. This can be done in a standard manner by adding and subtracting

$$
\frac{1}{32 \pi^{2}}\left(4 \pi \mu^{2}\right)^{-\epsilon} \int_{0}^{\infty} \frac{d s}{s^{3-\epsilon}}\left(1+\frac{s^{2}}{6} \omega_{\alpha}^{\alpha}\right) e^{-s M^{2}}
$$

It is not necessary to renormalize the term proportional to $\omega^{\alpha}{ }_{\alpha}$ since it is finite after a partial integration (the divergent part is a total derivative). We add it anyway to make $\mathcal{L}^{(1)}(x)$ finite before any partial integrations. The regularized expression in $4-2 \epsilon$ dimensions is then

$$
\begin{aligned}
\mathcal{L}^{(1)}(x)= & \frac{1}{32 \pi^{2}} \int_{0}^{\infty} \frac{d s}{s^{3}}\left[\operatorname{det}\left(\frac{s \sqrt{2 \omega}}{\sin (s \sqrt{2 \omega})}\right)^{1 / 2}\right. \\
& \left.\times \exp \left\{s F \frac{1}{2 \omega}\left(1-\frac{2}{s \sqrt{2 \omega}} \tan \left(\frac{s \sqrt{2 \omega}}{2}\right)\right) F\right\}-\left(1+\frac{s^{2}}{6} \omega^{\alpha}{ }_{\alpha}\right)\right] e^{-s M^{2}} \\
& +\frac{M^{4}}{64 \pi^{2}}\left(\frac{1}{\epsilon}+\frac{3}{2}-\gamma-\ln \frac{M^{2}}{4 \pi \mu^{2}}\right)-\frac{1}{32 \pi^{2}} \omega^{\alpha}{ }_{\alpha} \ln \frac{M^{2}}{4 \pi \mu^{2}} .
\end{aligned}
$$

Even though Eq. (16) looks manifestly real, there might be some imaginary contributions from poles on the real $s$-axis, depending on the eigenvalues of $\omega$. The contour should go slightly above those poles.

At this point it is interesting to start to series-expand Eq. (16) in derivatives to see if it coincides with known results. We then find before any partial integration:

$$
\begin{aligned}
\mathcal{L}^{(1)}(x) & \simeq \frac{M^{4}}{64 \pi^{2}}\left(\frac{1}{\epsilon}+\frac{3}{2}-\gamma-\ln \frac{M^{2}}{4 \pi \mu^{2}}\right)-\frac{1}{32 \pi^{2}} \frac{\omega^{\mu}{ }_{\mu}}{6} \ln \frac{M^{2}}{4 \pi \mu^{2}}-\frac{1}{32 \pi^{2}} \frac{F^{\mu} F_{\mu}}{M^{2}} \\
& +\frac{1}{16 \pi^{2}}\left[\frac{\left(F_{\mu} F^{\mu}\right)^{2}}{96 M^{8}}-\frac{F_{\mu} \omega^{\mu}{ }_{\nu} F^{\nu}}{60 M^{6}}-\frac{F_{\mu} F^{\mu} \omega^{\nu}{ }_{\nu}}{72 M^{6}}+\frac{\omega^{\mu}{ }_{\mu} \omega^{\nu}{ }_{\nu}}{144 M^{4}}+\frac{\omega^{\nu}{ }_{\mu} \omega^{\mu}{ }_{\nu}}{180 M^{4}}\right] .
\end{aligned}
$$

The effective potential (no derivatives) and the wave function renormalization (two derivatives) come out correctly up to partial integration. However, comparing with e.g. [4] or [5] one finds an apparent discrepancy for the four-derivative term (in [5] there is also an overall sign error). The difference is a total derivative up to a term which contains a field with three derivatives:

$$
\frac{1}{180}\left(\frac{\left(\omega_{\mu}^{\mu}\right)^{2}}{M^{4}}-\frac{2 F^{\mu} F_{\mu} \omega_{\nu}^{\nu}}{M^{6}}\right)=\partial^{\mu}\left(\frac{F_{\mu} \omega_{\nu}^{\nu}}{180 M^{4}}\right)-\frac{F_{\mu} \partial^{\mu} \omega^{\nu}{ }_{\nu}}{180 M^{4}} .
$$

In the present approximation we neglect the last term in Eq. (18) so that the result here is actually consistent with [4, 5]. This observation reminds us that the local effective Lagrangian density in the effective action is not uniquely defined since we can add total derivatives without changing the equations of motion. Only the integral over space-time or the equations of motion have intrinsic meaning. 


\section{ZERO FIELD LIMIT FOR $m^{2}=0$}

At the end of Section 2 we saw what a typical higher order term in the derivative expansion looks like (see Eq. (17)). It is easy to see that, for dimensional reasons, the higher the power of derivatives is, the higher the power of $M^{2}$ in the denominator is. For a massless theory, $M^{2}=\lambda \phi^{2} / 2$, so for small $\phi$ this expansion obviously breaks down. In the derivative expansion, corrections to derivative terms are computed assuming that the field is constant. Since the corrections diverges we have to resum the series. After all, if $\partial_{\mu} \phi$ is non-zero then $\phi$ cannot be zero everywhere. The propagator we calculated in Section 2 contains the resummation necessary to make the $\phi \rightarrow 0$ limit meaningful. It does not really make sense to talk about $\phi=0$ in the effective action since for instance $\partial_{\mu} \phi \partial^{\mu} \phi$ can be partially integrated to $\phi \partial_{\mu} \partial^{\mu} \phi$, which would then be zero although it does contribute to the equation of motion. In order to avoid the problems of possible partial integration and mixing of derivatives we shall study the equations of motion directly. From Eq. (4) we have the unrenormalized equation of motion

$$
\frac{\delta \Gamma_{c l}}{\delta \phi}-\frac{\lambda \phi(x)}{2} \int_{0}^{\infty} d s\langle x(s) \mid x(0)\rangle=0 .
$$

It may at first look strange that the whole one-loop contribution in Eq. (19) (using Eq. (13)) comes from varying Eq. (5) (using Eqs. (6, 14)) only with respect to the $\phi$ in $M^{2}$ and not in $F$ or $\omega$. But, rewriting Eq. (5) as

$$
\Gamma_{c l}[\phi]-\frac{i}{2} \int_{0}^{\infty} \frac{d s}{s} e^{-i s M^{2}(x)} \int d^{4} y\left\langle y\left|\exp \left[i s\left(\hat{p}^{2}-M^{2}(\hat{y})+M^{2}(x)\right)\right]\right| y\right\rangle,
$$

one sees that the linear variation with respect to $\phi$ only gets contributions from the first factor $e^{-i s M^{2}}$ and not from the operator part in $M^{2}(\hat{y})$. Partial integrations in Eq. (5) would, of course, spoil this property. Also the truncation of including only the second derivative, as in Eq. (14), spoils this property in general. We can verify explicitly from Eq. (17) that up to two derivatives

$$
\frac{\delta \Gamma}{\delta M^{2}}=\frac{\partial \mathcal{L}}{\partial M^{2}}-\partial_{\mu} \frac{\partial \mathcal{L}}{\partial F_{\mu}}+\partial_{\nu} \partial_{\mu} \frac{\partial \mathcal{L}}{\partial \omega_{\nu \mu}}=\frac{\partial \mathcal{L}}{\partial M^{2}},
$$

so that is does not matter whether it is only $M^{2}$ that is varied with respect to $\phi$ or also $F$ and $\omega$. In the case of four or more derivatives Eq. (21) no longer holds since for instance a term like $\partial^{4} M^{2} / M^{2}$ would contribute to the equations of motion in the present approximation, but it is not included in the effective Lagrangian in Eq. (14). This shows again that one should discuss the equations of motion where these ambiguities do not occur. 
To simplify the analysis we shall now assume that the background field is static and that the eigenvalues of $\omega_{i j}$ are positive. This is natural since $M^{2}=\lambda \phi^{2} / 2$ is positive for real $\phi$ and has to be growing (or constant) in all directions away from a point where $\phi=0$. We continue to suppress the space-time indices but in this section they should be understood as only space indices with Euclidean metric. Thus, we define $F$ and $\omega$ by $F_{i}$ and $\omega_{i j}$, with all indices as subscripts, so there is a sign difference with $\omega^{\mu}{ }_{\nu}$. In order to do a series expansion of Eq. (19) in powers of $\phi$ we write the one-loop part, after renormalization, as

$$
\begin{aligned}
\Gamma^{(1)}(x)=- & \frac{\lambda \phi}{32 \pi^{2}} \int_{0}^{\infty} \frac{d s}{s^{2}}\left[\operatorname{det}\left(\frac{s \sqrt{2 \omega}}{\sinh (s \sqrt{2 \omega})}\right)^{1 / 2}\right. \\
& \times\left(\exp \left\{s \lambda^{2} \phi^{2} \partial_{i} \phi\left(\frac{1}{2 \omega}\left(1-\frac{2}{s \sqrt{2 \omega}} \tanh \left(\frac{s \sqrt{2 \omega}}{2}\right)\right)\right)_{i j} \partial_{j} \phi\right\}-1\right) \\
& \left.-\left(1-\operatorname{det}\left(\frac{s \sqrt{2 \omega}}{\sinh (s \sqrt{2 \omega})}\right)^{1 / 2}\right)\right] \exp \left(-s \frac{\lambda \phi^{2}}{2}\right) \\
& +\frac{\lambda \phi}{32 \pi^{2}} \frac{\lambda \phi^{2}}{2}\left(\frac{1}{\epsilon}+1-\gamma-\ln \frac{\lambda \phi^{2}}{8 \pi \mu^{2}}\right) .
\end{aligned}
$$

The two terms in the square brackets are separately finite for small $s$, and we shall treat them separately. The first one can actually be expanded directly in $\phi$ since the determinant makes the integral convergent for large $s$. The second term has to be treated with more care. One way to evaluate it is to divide the $s$-integral into two intervals, $[0, \Lambda]$ and $[\Lambda, \infty]$, where $\lambda \phi^{2} \ll \Lambda^{-1} \ll\|\omega\|^{1 / 2}$. Doing suitable approximations and partial integrations on each interval, we finally obtain

$$
\begin{aligned}
\Gamma^{(1)}(x) \simeq-\frac{\lambda \phi}{32 \pi^{2}} & \left\{\lambda^{2} \phi^{2} \int_{0}^{\infty} \frac{d s}{s} g(s) \partial_{i} \phi\left[\frac{1}{2 \omega}\left(1-\frac{2}{s \sqrt{2 \omega}} \tanh \left(\frac{s \sqrt{2 \omega}}{2}\right)\right)\right]_{i j} \partial_{j} \phi\right. \\
& \left.-\int_{0}^{\infty} \frac{d s}{s^{2}}(1-g(s))+\frac{\lambda \phi^{2}}{2}\left(-\frac{1}{\epsilon}+\int_{0}^{\infty} d s \ln \left(4 \pi \mu^{2} s\right) \frac{d g(s)}{d s}\right)\right\},
\end{aligned}
$$

where

$$
g(s) \equiv \operatorname{det}\left(\frac{s \sqrt{2 \omega}}{\sinh (s \sqrt{2 \omega})}\right)^{1 / 2} .
$$

It is interesting to see that Eq. (23) has a well-defined and finite expansion in powers of $\phi$. The reason why it is IR-finite, i.e. why it is convergent for large $s$, is that $g(s) \rightarrow 0$ rapidly. We do not see immediately from the treatment here how higher order derivatives of $M^{2}$ would affect the result, but we can expect the correction to be finite on physical grounds if there are no instabilities. 


\section{Finite temperature efFective aCtion}

There are two main formalisms for doing finite temperature field theory, the real-time and the imaginary-time formalisms, and both can be used to generalize the calculations in Section 2. We shall discuss both formalisms here and use them for different purposes.

\section{REAL-TIME FORMALISM}

The generalization to the real-time formalism is done by using the thermal propagator when calculating the tadpole in Eq. (田). Although a doubling of the number of degrees of freedom is generally needed for a consistent loop expansion, only the (11)-part of the propagator is needed to calculate the tadpole at one loop. The thermal (11)-propagator is given by

$$
\frac{i}{p^{2}-M^{2}+i \epsilon}+2 f_{B}\left(p_{0}\right) \operatorname{Re} \frac{i}{p^{2}-M^{2}+i \epsilon} .
$$

The real part can be represented by extending the $s$-integral in Eq. (6) from $-\infty$ to $\infty$, but the phase of the normalization in Eq. (13) is only valid for $s>0$. Instead of keeping track of the phase factor we continue with explicitly taking the real part. The order of $f_{B}\left(p_{0}\right)$ and $M^{2}(\phi(\hat{x}))$ in Eq. (25) is not well-defined, unless the background field is time-independent. It also makes sense to limit the considerations to such fields since the system will not remain in equilibrium otherwise. It is conceivable to treat small time-dependent perturbations of an equilibrium background, assuming the perturbation to be small, but here we also want to deal with large field amplitudes. Thus, assuming $F_{0}=0$ and $\omega_{\mu 0}=0$, we get

$$
\begin{aligned}
\mathcal{L}^{\beta, \mu}(\phi)= & -\operatorname{Re} \int \frac{d p_{0}}{2 \pi} f_{B}\left(p_{0}\right) e^{-i \frac{\pi}{4}} \int_{0}^{\infty} \frac{d s}{s}(4 \pi s)^{-3 / 2} \\
& \times\left[\operatorname{det}\left(\frac{s \sqrt{2 \omega}}{\sin (s \sqrt{2 \omega})}\right)^{1 / 2} \exp \left[i F \frac{s}{2 \omega}\left(1-\frac{2}{s \sqrt{2 \omega}} \tan \left(\frac{s \sqrt{2 \omega}}{2}\right)\right) F\right]-1\right] \\
& \times \exp \left[i s\left(p_{0}^{2}-M^{2}\right)\right] \\
& +\frac{1}{12 \pi^{2}} \int d p_{0} f_{B}\left(p_{0}\right)\left(p_{0}^{2}-M^{2}\right)^{3 / 2} \Theta\left(p_{0}^{2}-M^{2}\right)
\end{aligned}
$$

The last term in Eq. (26) is the effective potential, which comes out when we regularize for small $s$ and use a $\Gamma$-function to define a finite value.

This formal expression of the thermal effective action is rather difficult to use in general, since we would like to deform the $s$-contour to the positive imaginary axis for large $p_{0}$, but then we encounter all the poles on the positive real axis (the $s$-contour as it stands goes slightly below the real axis). At least if we assume the eigenvalues of $\omega_{i j}$ 
to be positive, which we want for stability. The situation is reminiscent of electrons in a constant magnetic field [6] where the poles correspond to Landau levels, while here they are related to oscillations in a harmonic oscillator potential.

As in Section 2, we want to compare Eq. (26) with known derivative expansions. The effective potential is easily recognized to be correct from the last term in Eq. (26). The wave function renormalization comes from two terms, $\omega_{i i}$ and $F_{i} F_{i}$. A naive expansion in $F_{i} F_{i}$ of the exponent in Eq. (26) leads to a divergent integral, but if one first makes a partial integration with respect to $p_{0}$ it becomes finite. In the end the total contribution agrees with the high temperature expansion in [5].

\section{IMAGINARY-TIME FORMALISM}

The transition to the imaginary-time formalism is most easily performed in a $p_{0}$ rather than $x_{0}$ representation of the amplitude $\left\langle x^{\prime \prime}(s) \mid x^{\prime}(0)\right\rangle$. In the limit of coinciding points we can write

$$
\langle x(s) \mid x(0)\rangle=\int \frac{d p_{0}}{2 \pi} d x_{0}^{\prime}\left\langle x_{0}^{\prime}(s), x_{i}(s) \mid x_{0}(0), x(0)_{i}\right\rangle e^{i p_{0}\left(x_{0}^{\prime}-x_{0}\right)},
$$

and replace $\int d p_{0}$ by $i 2 \pi T \sum_{n}\left(p_{0} \rightarrow i 2 \pi T n\right)$, as usual. Let us now study the equation of motion and see what happens in the $\phi \rightarrow 0$ limit when $m^{2}=0$. [ The full one-loop correction to the equation of motion is

$$
\begin{aligned}
\Gamma^{(1)}[\phi] & +\Gamma_{\beta}^{(1)}[\phi]=-\frac{\lambda \phi}{2} \int_{0}^{\infty} d s e^{-i \frac{\pi}{4}} T \sum_{n=-\infty}^{\infty}(4 \pi s)^{-3 / 2} \operatorname{det}\left(\frac{s \sqrt{2 \omega}}{\sin (s \sqrt{2 \omega})}\right)^{1 / 2} \\
& \times \exp \left[-i s\left((2 \pi T n)^{2}+M^{2}-F \frac{s}{2 \omega}\left(1-\frac{2}{s \sqrt{2 \omega}} \tan \left(\frac{s \sqrt{2 \omega}}{2}\right)\right) F\right)\right] .
\end{aligned}
$$

We shall only study the leading contribution for small $\phi$, so we can put $M^{2}=F=0$. The IR convergence is anyway governed by $\omega$. The zero temperature part of Eq. (28) can be extracted after a Poisson resummation, and in the remaining piece we deform the contour by taking $s \rightarrow-i s$. Thus the finite value of $\Gamma_{\beta}^{(1)}$ for small $\phi$ is

$$
\Gamma_{\beta}^{(1)}[\phi] \simeq-\frac{\lambda \phi}{16 \pi^{2}} \int_{0}^{\infty} \frac{d s}{s^{2}} \operatorname{det}\left(\frac{s \sqrt{2 \omega}}{\sinh (s \sqrt{2 \omega})}\right)^{1 / 2} \sum_{n=1}^{\infty} \exp \left(\frac{-n^{2}}{4 T^{2} s}\right)
$$

To get some more concrete information out of Eq. (29) we can take the high temperature limit where the determinant can be approximated by 1 . As expected we find

\footnotetext{
${ }^{2}$ To be consistent in the high temperature limit we would have to resum the thermal mass correction $\lambda T^{2} / 24$. A zero effective mass is found at a second order phase transition in a spontaneously broken theory where the resummed mass is $m^{2}(T)=-\mu^{2}+\lambda T^{2} / 24, \mu^{2}>0$. This is really the situation we imagine when we write $m^{2}=0$ here.
} 
$\Gamma_{\beta}^{(1)}=-\lambda T^{2} \phi / 24$, which simply is the thermal mass shift. Therefore, we also want the next to leading term in order to get something new. Again, a straightforward expansion of the determinant does not work since it gives divergent remaining integrals. This is not surprising since it is the large- $s$ behaviour of the determinant that should make the expansion IR-convergent. One way to get around the problem is to first subtract the 1 from the determinant and then use the formula

$$
\sum_{n=1}^{\infty} e^{-n^{2} / s}=\frac{1}{2}(\sqrt{\pi s}-1)+\sqrt{\pi s} \sum_{n=1}^{\infty} e^{-n^{2} \pi^{2} s}
$$

and a transformation $s \rightarrow 1 / s$ to restrict the integration interval to $[1, \infty]$. We then obtain for the subleading contribution in $T$

$$
\begin{aligned}
\Gamma_{\beta}^{(1)}[\phi]+\frac{\lambda T^{2} \phi}{24}= & -\frac{\lambda \phi T^{2}}{4 \pi} \int_{1}^{\infty} d s\left[\left(g\left(\frac{1}{4 \pi T^{2} s}\right)-1\right)+s^{-3 / 2}\left(g\left(\frac{s}{4 \pi T^{2}}\right)-1\right)\right] \sum_{n=1}^{\infty} e^{-n^{2} \pi s} \\
& -\frac{\lambda \phi T^{2}}{8 \pi^{2}} \int_{1}^{\infty} d s\left(g\left(\frac{s}{4 \pi T^{2}}\right)-1\right)\left(s^{-3 / 2}-s^{-2}\right)
\end{aligned}
$$

using the notation in Eq. (24). The first term on the right-hand side of Eq. (31) goes to zero in the high temperature limit, but the second term finally gives:

$$
\Gamma_{\beta}^{(1)}[\phi] \simeq-\frac{\lambda T^{2}}{24} \phi-\frac{\lambda T}{16 \pi^{3 / 2}} \phi \int_{0}^{\infty} \frac{d s}{s^{3 / 2}}\left[\operatorname{det}\left(\frac{s \sqrt{2 \omega}}{\sinh (s \sqrt{2 \omega})}\right)^{1 / 2}-1\right]
$$

We can then conclude that also the finite temperature part of the equation of motion is well-behaved when $\phi \rightarrow 0$ as long as $\omega$ has positive eigenvalues.

\section{References}

[1] J. Schwinger, "On gauge invariance and vacuum polarization", Phys. Rev. 82 (1951) 664 .

[2] D.-U. Jungnickel and D. Walliser, "Inhomogeneous field configurations and the electroweak phase transition", Phys. Rev. D49 (1994) 3869,

D. Bödeker, W. Buchmüller, Z. Fodor and T. Helbig, "Aspects of the cosmological electroweak phase transition", Nucl. Phys. B423 (1994) 171,

J. Kripfganz, A. Laser and M. G. Schmidt, "Critical bubbles and fluctuations at the electroweak phase transition", Nucl. Phys. B433 (1995) 467.

[3] P. Elmfors, K. Enqvist and I. Vilja, "Finite temperature effective action and thermalization of perturbations", Nucl. Phys. B422 (1994) 521. 
[4] C. M. Fraser, Calculation of higher derivative terms in the one-loop effective Lagrangian”, Z. Phys. C28 (1985) 101.

[5] I. Moss, D. Toms and A. Wright, "Effective action at finite temperature", Phys. Rev. D46 (1992) 1671.

[6] P. Elmfors, D. Persson and B.-S. Skagerstam, "Real-time thermal propagators and the QED effective action for an external magnetic field", Astropart. Phys. 2 (1994) 299. 